\title{
NUMERICAL AND EXPERIMENTAL INVESTIGATIONS ON THE CAVITATING FLOW IN A CASCADE OF HYDROFOILS
}

\author{
H. Lohrberg, B. Stoffel \\ Laboratory for Turbomachinery and Fluid Power, Darmstadt University of Technology \\ R. Fortes-Patella, JL. Reboud (*) \\ Laboratoires des Ecoulements Géophysiques et Industriels, Grenoble, France \\ ${ }^{(*)}$ LTDS / ENISE, Saint Etienne, France
}

\begin{abstract}
The cavitating flow in a cascade of three hydrofoils was investigated by experimental means and numerical simulation. Experiments on the 2D-hydrofoils cascade were carried out at Darmstadt University of Technology in a rectangular test section of a cavitation tunnel. A numerical model developed at LEGI (Grenoble) to describe the unsteady behaviour of cavitation including the shedding of vapour structures was applied to the hydrofoils cascade geometry. Results of both experimental and numerical studies show a strong interaction between the cavities of each flow channel besides the typical self-oscillation of cloud cavitation. A detailed comparison of the results allows proposing an interpretation of the interaction mechanisms.
\end{abstract}

\section{Nomenclature}

\begin{tabular}{|c|c|c|c|}
\hline $\mathrm{Cp}$ & : pressure coefficient & $=(\mathrm{P}-$ Pref $) /\left(1 / 2 \rho_{1} \operatorname{Vref}^{2}\right)$ & $(-)$ \\
\hline $\mathrm{f}$ & : frequency & & $(\mathrm{Hz})$ \\
\hline Lcav & : cavity length & & (m) \\
\hline Lref & : reference length & $=$ chord of the foil $=5 \mathrm{~cm}$ & (m) \\
\hline Vref & : reference velocity & $=$ inlet flow velocity & $(\mathrm{m} / \mathrm{s})$ \\
\hline $\mathrm{P}$ & : static pressure & & $(\mathrm{Pa})$ \\
\hline Pref & : reference pressure & $=$ inlet static pressure & (Pa) \\
\hline $\mathrm{Pv}$ & : vapour pressure & & $(\mathrm{Pa})$ \\
\hline St & : Strouhal number & $=\mathrm{f}$ Lcav $/$ Vref & $(-)$ \\
\hline Tref & : reference time & $=$ Lref $/$ Vref & (s) \\
\hline$\sigma$ & : cavitation number & $=(\operatorname{Pref}-\mathrm{Pv}) /\left(1 / 2 \rho_{l} \operatorname{Vref}^{2}\right)$ & $(-)$ \\
\hline$\rho_{1}$ & : liquid density & & $\left(\mathrm{kg} / \mathrm{m}^{3}\right)$ \\
\hline
\end{tabular}

\section{Introduction}

In the scope of the European Research Program PROCOPE, researchers of the LEGI (Laboratoire des Ecoulements Géophysiques et Industriels de Grenoble), and of the TUD (Laboratory for Turbomachinery and Fluid Power of Darmstadt) work together in order to improve the understanding of the unsteady behaviour of cavitating flows and the related erosive aggressiveness. In this context, experimental investigation and numerical modelling of cavitating flows around a 2D-hydrofoil with a semicircular nose were performed. Previous results concerning the self-oscillating behaviour of cloud cavitation were presented in [Hofmann et al. 1999], and the stabilising effect of a small obstacle modifying the foil suction side has been more particularly studied. In the present paper, the cavitating flow in a cascade of three hydrofoils was investigated by experimental means and numerical simulation.

Experiments on the 2D-hydrofoils cascade were carried out at Darmstadt University of Technology in a rectangular test section of a cavitation tunnel. Transient wall pressure signals were measured, treated to evaluate the dominant frequencies and used to perform conditional sampling of images of the cavitating flow. Images of the cavitating flow were taken synchronously with the pressure transients to provide further information about the cavitation dynamics. 
Numerical models of unsteady cavitating flows using single fluid approaches have been developed last years. In such models, the same velocity is assumed for liquid and vapour phases in the two-phase zones. Vaporisation and condensation of the fluid are managed by using barotropic state law, mass transfer equation or bubble dynamic model. Besides applications of different models to quasi-steady cavitating flow, some studies focused on unsteady cloud cavitation phenomenon in Venturi type ducts [Delannoy and Kueny, 1990, Reboud et al, 1998] or around hydrofoils in cavitation tunnels [Kubota et al. 1992, Reboud and Delannoy, 1994, Song and He, 1998, Sauer and Schnerr 2000]. Numerical simulation of the stabilising effect of small obstacle placed in the cavitation sheet area [Hofmann et al. 1999], shows the ability of such model to catch localised hydrodynamic effects. Recent extension to blade cascades representative of inducer geometries [Coutier-Delgosha et al. 2000], allows to predict instabilities due to presence of sheet cavitation on the different blades, showing a reliable agreement with experimental observations [Joussellin et al. 2001].

In the present paper, the numerical model developed at LEGI (Grenoble) to describe the unsteady behaviour of cavitation including the shedding of vapour structures, was applied to the hydrofoils cascade geometry. Comparison of experimental and numerical results are performed to analyse the interaction between the cavitating flows in the different channels.

\section{Experimental Set-up}

Object of the investigations is a cascade of three hydrofoils, located in a test section with a rectangular cross section of 30 by 100 millimetres (Figure 1). The hydrofoils can be adjusted to different angles of attack in a range of $\pm 10^{\circ}$ according to different flow rates in a centrifugal pump. The flow can be illuminated and observed through a lateral window. Illumination war done with a stroboscopic light source, images were taken with a CCD-camera (PCO sensicam). A piezoresistive pressure transducer (Kulite XTM-190M) with a frequency range up to $125 \mathrm{kHz}$ was mounted in the window too. The membrane is connected to the flow by a small, short opening. Geometry of the hydrofoils and sensor position are shown in figure $1 \mathrm{~b}$. The sensor is mounted above profile 1, the positions above profile 2 and 3 are used for post-processing of the numerical results. The test section is part of a closed hydraulic loop. Flow rate and static pressure can be varied in order to achieve different cavitation conditions. In the case presented hereafter, the hydrofoils are held at 5 degree angle of attack, and the inlet velocity Vref is equal to $14 \mathrm{~m} / \mathrm{s}$.

Image illumination and acquisition and pressure signal recording were both triggered by a personal computer. Signals were sampled with $100 \mathrm{kHz}$ and stored in a transient recorder. Storing started with $50 \%$ pre-trigger, thus the image was taken in the middle of the measurement. Images and measurements were read out and stored on a personal computer. Every image is then related to a part of the pressure signal.

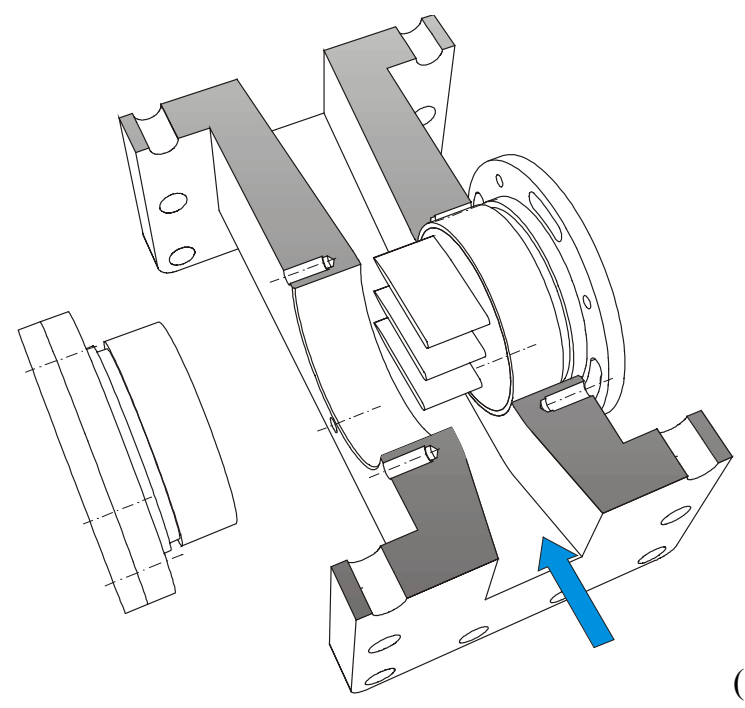

(a)

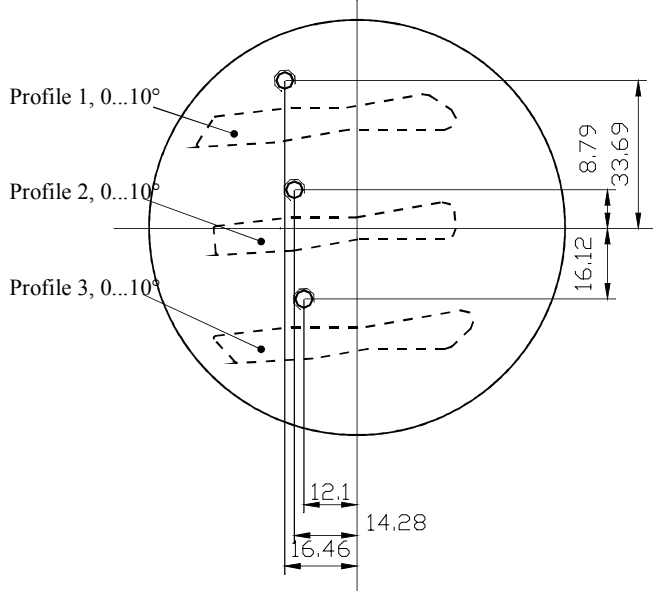

Figure 1. Hydrofoils cascade geometry - a) Cut through the test section (pressure transducers are not shown), b) side view of the window with one sensor (upper channel) and three post-processing positions. 


\section{Signal treatment}

First the power density spectra of the signals were calculated. They showed two dominant frequencies for each signal. To extract those frequencies from the very noisy pressure signal, a more careful method was applied to the signals.

First the signals were split at $100 \mathrm{~Hz}$ into high and low frequent parts by high and low pass filtering respectively. The auto-correlation function was then calculated form both parts. The function of a fully periodic signal would show several peaks with a maximum of one. The first is located at zero shifting, the others at positive and negative multiples of cycle duration. Due to the variation of the frequency of a real signal these peaks do not have a maximum of one. If the variation is seen as a phase difference between successive periods, the maximum value of the first peak next to the center is the cosine of this phase difference. The frequency variation can therefore be calculated from the peak value at cycle duration. The both frequencies and their variation are shown as a function of the cavitation number $\sigma$ in Figure 2 .

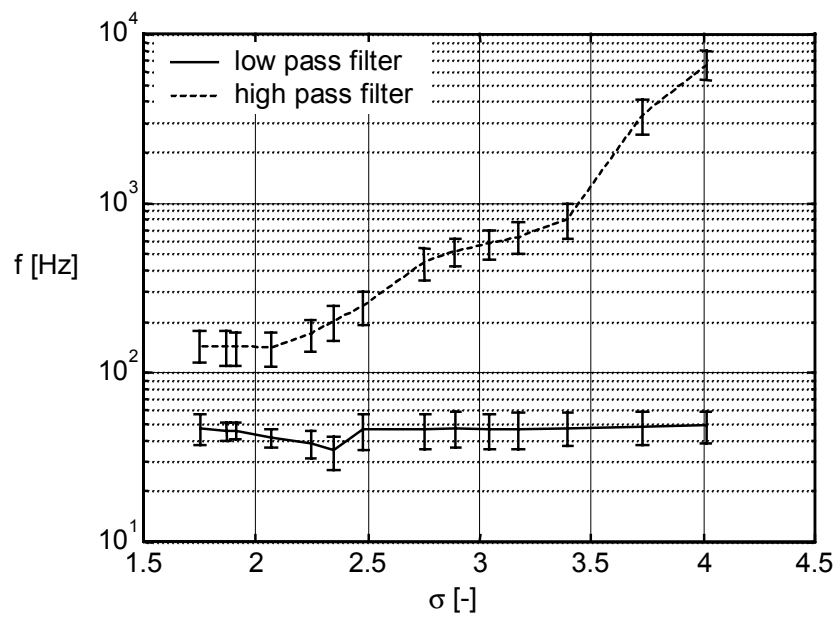

Figure 2. Main frequency of the low and high pass filtered pressure signal as a function of the cavitation number $\sigma, \operatorname{Vref}=14 \mathrm{~m} / \mathrm{s}$.

The higher frequencies correspond to the cavitation self-oscillation behaviour, characterised by Strouhal Number between 0.2 and 0.3 . The lower frequency was presumed to be related to a coupling of the cavitation on the three hydrofoils. To verify this presumption, images were associated to the phase angle of the low frequency fluctuation.

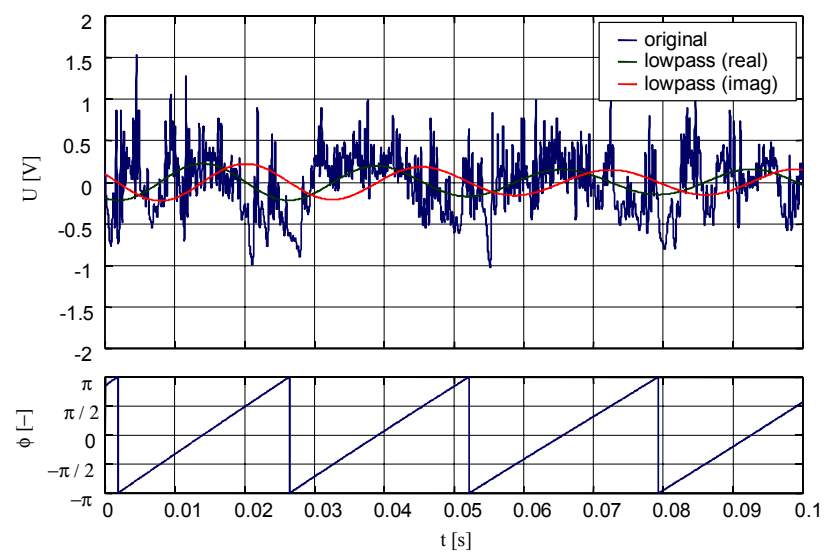

Figure 3. Raw pressure signal, low pass filtered and Hilbert transformed signal, phase angle.

This fluctuation was extracted by filtering the original signal. The filter design based on the auto-correlation function of the signal. The mean cycle frequency plus / minus the frequency variation are taken as the edge 


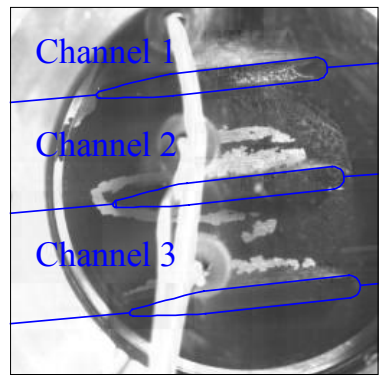

$\varphi=0$

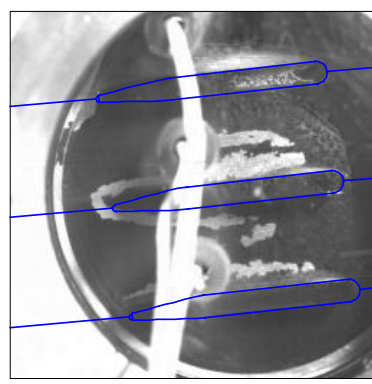

$\varphi=1 / 2 \pi$

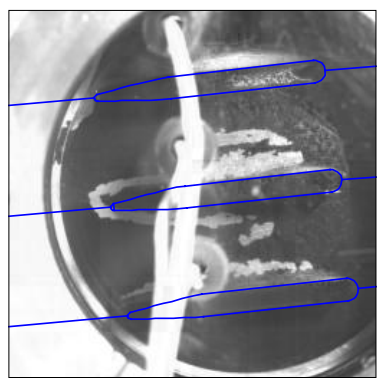

$\varphi=\pi$

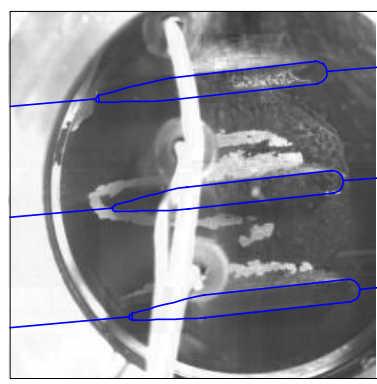

$\varphi=3 / 2 \pi$

Figure 4. Exemplary frames from a sequence of phase angle averaged images, $\alpha=5^{\circ}, \sigma=1.9$, Vref $=14 \mathrm{~m} / \mathrm{s}$.

frequencies of a band pass filter. Filtering was carried out by Fourier transformation, deleting of the parts with frequencies outside the band and inverse Fourier transformation. By this, no further delay was added to the signal.

After filtering the signals were undertaken a Hilbert transformation. This transformation adds an imaginary part to the signal by shifting the Fourier coefficients by an angle of $\pi / 2$ and performing the inverse Fourier transformation. The phase information can be extracted from the complex signal (Figure 3). The nonconsecutively acquired images can then be sorted into phase angle consecutive order.

With the phase information available for every image, there are two further treatments possible both used to explain the cavitation behaviour and compare the experimental and the numerical results respectively.

First, the images were put together to an exemplary sequence of one cavitation cycle (Figure 4). The cycle was divided into 25 steps. On the basis of the phase angle, every image was assigned to one step. With a total image number of one thousand, every step includes about forty images. These images were averaged by a special algorithm in order to let the resulting image appear like a real one shot cavitation image. This approach makes the subsequent interpretation of the sequence easier. A wavelet transformation was applied using sigmoid wavelets. The wavelet coefficients were then averaged by absolute value and sign thus keeping the image signals energy in every wavelet form. The inverse transformation was applied on the averaged coefficients.

The second treatment extracted the mean brightness of three regions of interest, each related to one channel of the cascade. As the vapour appears bright in the image, the mean brightness is a measure of the vapour volume even if the relation between both is not quantitatively known. The mean grey values are normalised by mean value and standard deviation. The first harmonic amplitude of the brightness is calculated. The harmonic functions of each channel are shown in Figure 5. The amount of the signals energy of the brightness that is stored in the first harmonic (value $e$ in the legend) is only about $15 \%$. That means, that only a small part of vapour variation is related to the low fluctuation cycle. The larger part is related to cloud shedding and noise.

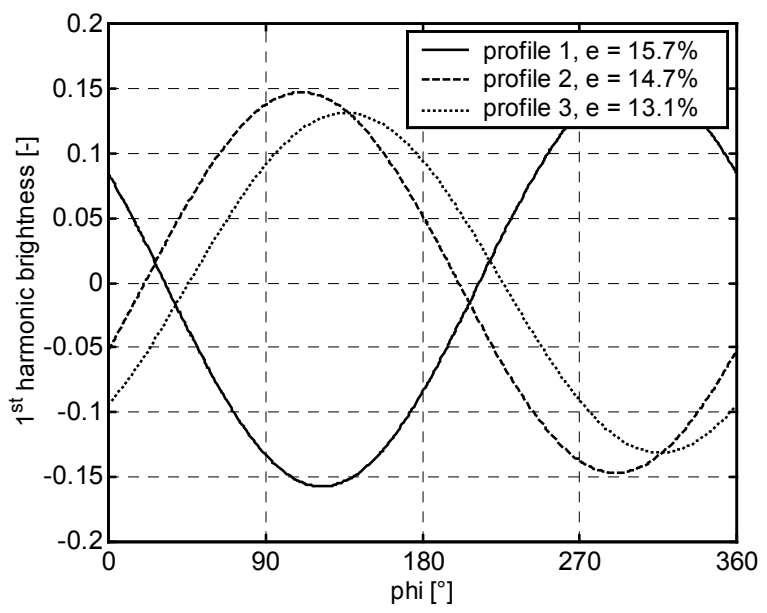

Figure 5. $1^{\text {st }}$ harmonic function of the brightness of each channel. 


\section{Numerical model}

To model and to analyse the unsteady behaviour of the cavitating flow in the tested hydrofoils cascade, the unsteady cavitating flow model developed at LEGI (Grenoble) [Delannoy and Kueny, 1990, Reboud and Delannoy, 1994, Reboud et al. 1998] was applied.

The theoretical and numerical model was developed to describe the unsteady behaviour of cavitation, including vapour clouds shedding. In that model, the liquid-vapour two-phase flow is described by homogeneous flow model with a no-slip condition between liquid and vapour phases. The liquid-vapour mixture, with a void ratio $\alpha$, is considered as a homogeneous medium and described as a single fluid, which varying specific mass is $\rho=\alpha$ $\rho_{\mathrm{v}}+(1-\alpha) \rho_{\mathrm{l}}$. A simple description of the vaporisation and condensation phenomena is proposed by introducing an empirical barotropic state law $\rho(\mathrm{p})$ for the mixture. A smooth law was chosen, $\rho$ rapidly varying between liquid density $\rho_{1}$ and vapour density $\rho_{v}$ when the local static pressure $P$ is around the vapour pressure $P v$. The law is characterised by its maximum slope at $\mathrm{P}=\mathrm{Pv}$, which is related to the minimum speed of sound $\mathrm{A}_{\min }$ in the two-phase homogeneous medium. $A_{\min }$ is the only adjustable parameter of the model, fixed in the present study as in the previous one [Hofmann et al., 1999] at 10\% of inlet velocity Vref.

Reynolds-averaged Navier-Stokes equations are solved in the case of this single fluid with variable density. A finite volume spatial discretisation is applied in curvilinear orthogonal co-ordinates on a staggered mesh.

An iterative resolution based on the SIMPLE algorithm was developed to deal with incompressible flow ( $\alpha=0$ and $\alpha=1)$ and highly compressible flow $(0<\alpha<1)$ [Delannoy and Kueny, 1990]. The liquid-vapour interfaces are described by high gradients of density, which was made possible by the use of a conservative approach and the HLPA non-oscillatory second order MUSCL scheme [Zhu, 1991]. To solve the time-dependant elliptic problem a first order fully implicit method is used. Turbulent flows are calculated by solving the Reynolds equations using a k- $\varepsilon$ RNG turbulence model with law of the wall.

The mass flow rate is imposed constant as upstream boundary condition. The transient static pressure, imposed as a downstream boundary condition, is slowly decreased from a high value, leading to steady non-cavitating conditions, to the value required to assure the expected cavitation number $\sigma$. Phase change then occurs spontaneously in the regions where the pressure decreases under the vapour pressure Pv. Because the experimental value of $\sigma$ is based on an upstream pressure measurement as reference pressure Pref, the losses generated in the cavitation tunnel by viscous and unsteady effects have to be taken into account in the calculation to compare a posteriori the experimental and numerical cavitation numbers.

The model was previously tested in Venturi type ducts [Reboud et al. 1998] and on single hydrofoil geometries [Reboud and Delannoy, 1994, Hofmann et al., 1999]. Empirical reduction of turbulence dissipative terms in the two-phase regions was proposed, to allow the simulation the unsteady behaviour of cavitation observed experimentally: dissipation in the pure liquid phase remains provided by the classical k- $\varepsilon$ RNG model, while stronger shear flows are allowed in the two phase regions by arbitrary reduction of the turbulence model constant $\mathrm{C}_{\mu}$ (multiplication by a function $\mathrm{f}(\rho)$, equal to 1 for $\rho=\rho_{1}$ and rapidly decreasing towards $\mu_{v} / \mu_{1}$ when $\rho<\rho_{1}$ ) [Reboud et al. 1998]. Effects on unsteady cavitation modelling of other modifications of standard turbulence models have been investigated recently and are reported in [Coutier-Delgosha et al. 2001].

\section{Simulation of the unsteady cavitating flow in the cascade}

In the case presented hereafter, the static pressures was decreased to the experimental value $\sigma=1.9$. Vapour structures, characterised in the model by small values of the density $\rho$, are generated along the suction side of the foils from the leading edge. After the initial transient, a special unsteady behaviour is observed (Figure 6):

- on the upper foil suction side, typical cloud cavitation behaviour takes place. However, the size and shedding period of the cavitation structures show strong variations between the successive cycles.

- on the two other foils the cavities appear as almost stable sheets attached to the blade, but with large amplitude and low frequency fluctuations of length.

The observed low frequency behaviour shows a period of $33 \mathrm{~ms}$, which is a frequency of $30 \mathrm{~Hz}$ with an uncertainty lower than $5 \%$. Frequency of cloud shedding process in the upper channel (named hereafter channel 1) can be estimated for the largest structures to $180 \mathrm{~Hz}+/-10 \%$. Based on the corresponding maximum attached cavity length, the Strouhal number is found to be about $0.27+/-10 \%$. The two values present a quite good agreement with the experimental values obtained from Figure 2 at $\sigma=1.95$ (respectively about 40-50hz, and $110-180 \mathrm{~Hz})$. 


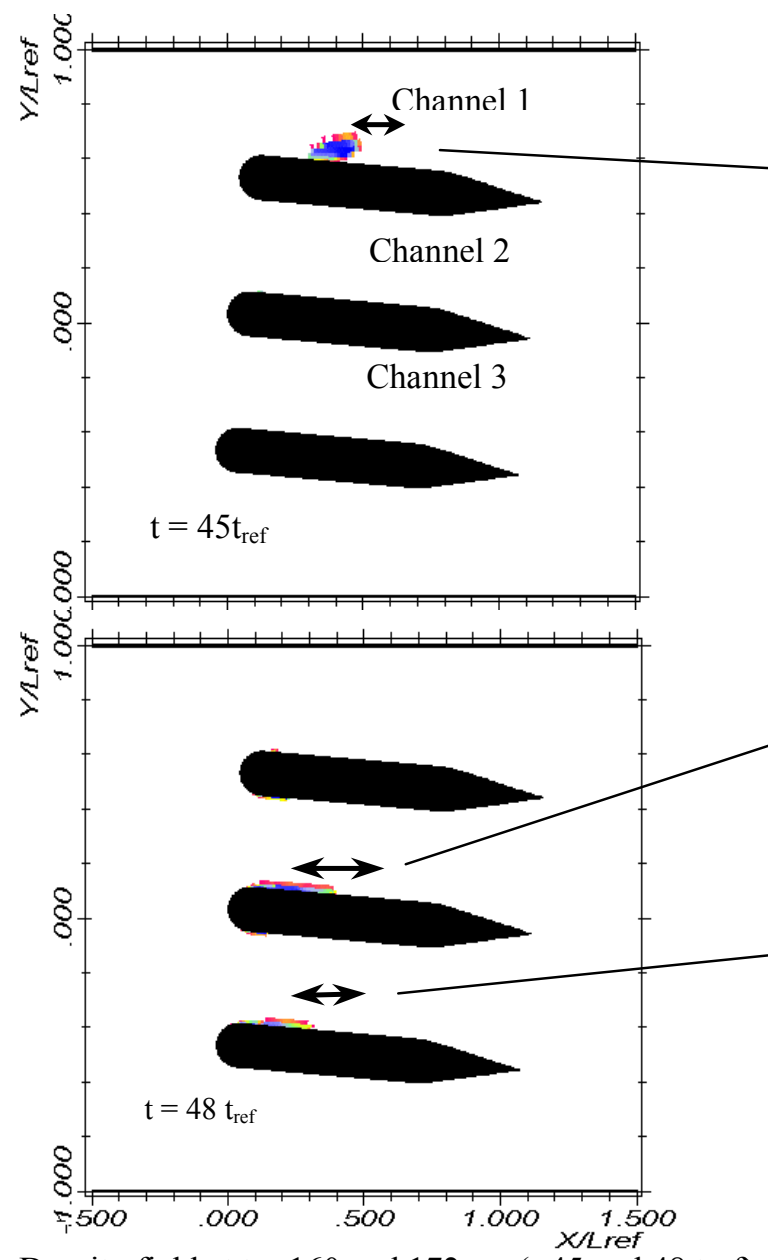

Density field at $\mathrm{t}=160$ and $172 \mathrm{~ms}$ ( $=45$ and 48 tref)

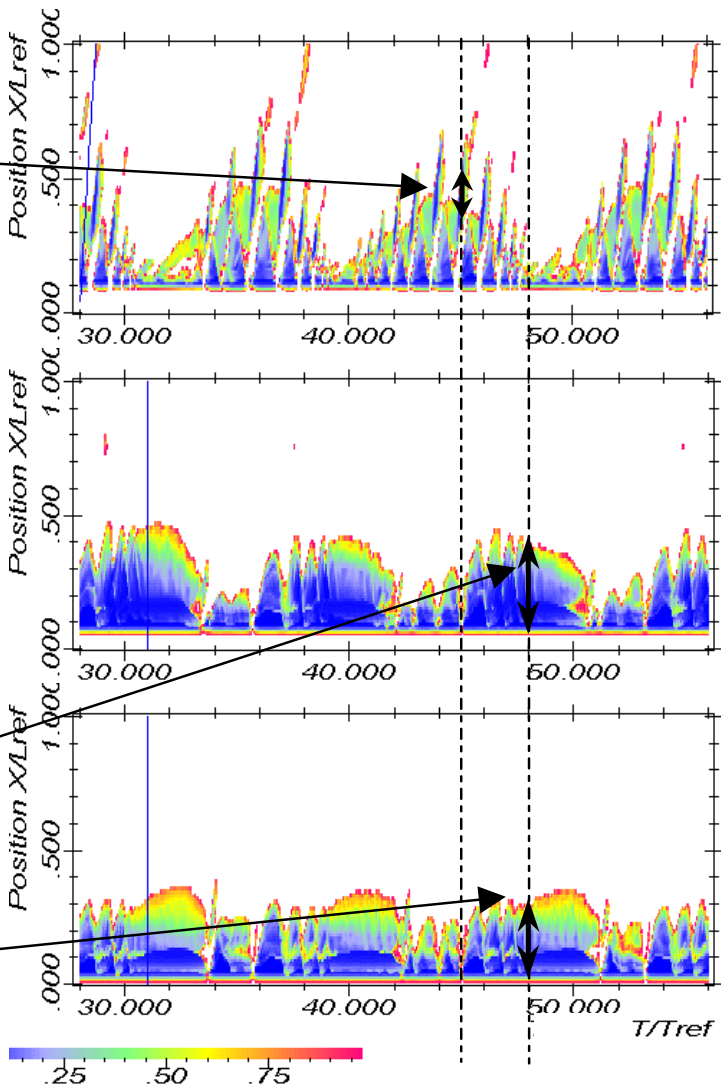

minimum value of density $\left(\rho / \rho_{l}\right)$

in cross sections of the channels.

Variation with time $($ Tref $=$ Lref $/$ Vref $=3.6 \mathrm{~ms})$ of the extend of the cavitation structures in the channels.

Figure 6. Time evolution of the cavitation structures on the three hydrofoils $($ Vref $=14 \mathrm{~m} / \mathrm{s}, \sigma=1.95)$

The difference between the cavitation behaviour in channel 1 and the others is associated to the larger angle of attack observed in front of the upper hydrofoil. This effect is due to the presence of the upper wall. The divergence angle between the wall and the foil suction side, associated with the trailing edge condition, increases the flow rate passing over the foil. An opposite effect occurs between the third foil and the lower wall. With the larger angle of attack, typical unsteady cloud cavitation takes place, while stable cavities with low relative thickness develop on the two other foils (channels 2 and 3). It can be seen in Figure 6, that the fluctuations of the cavity extend (as cloud cavitation in channel 1 or attached sheet cavitation in channels 2 and 3) appear completely out of phase. Disappearance of the cavitation structures in the upper channel correspond to a maximum development of the cavitation sheets on the two other foils, and vice versa.

Transient pressure coefficient distributions corresponding to three locations (Figure 1) are given in Figure 7, while two pressure fields are given at two different times of the low frequency cycle (Figure 8).

Larger development of cloud cavitation in channel 1 is associated with high-pressure fluctuations. The amplitude is higher in the first channel, because of the travelling clouds passing the point corresponding to the transducer location, but pressure fluctuations can also be clearly noticed in the two other channels. Contrarily, the phases during which cavitation is more developed as stable sheets on the two other foils show a complete stabilisation of the pressure signal.

Figure 9 shows the time variation of the vapour volume computed in the three channels. The out of phase behaviour between channel 1 and the two others is clearly visible. A small phase shift between the channels 2 and 3 may be noticed too. Those behaviours are in total agreement with the analysis of Figure 5, showing the phase shifts between the brightness fluctuations in the different channels. Despite the observed different 


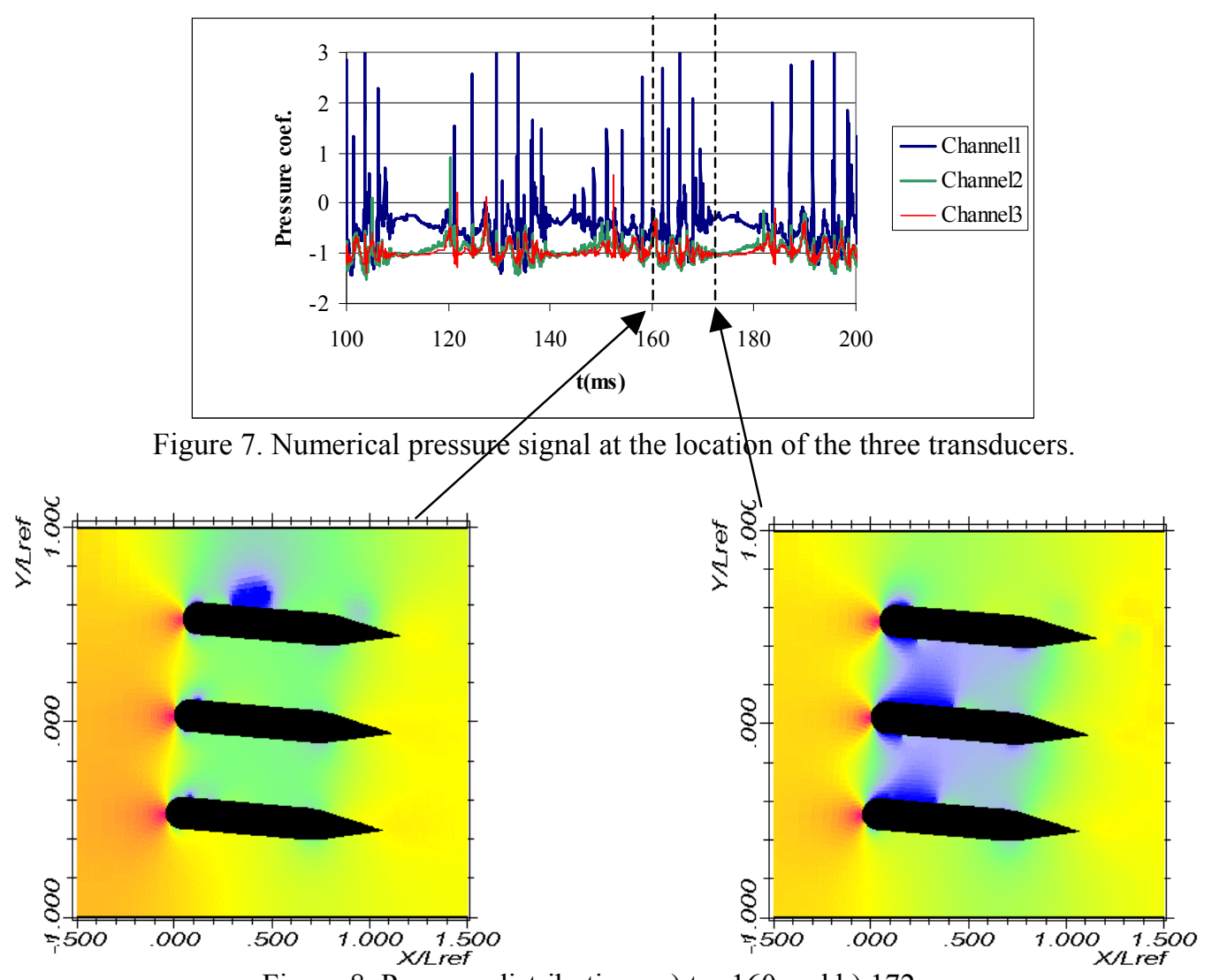

Figure 8. Pressure distribution : a) $\mathrm{t}=160$ and b) $172 \mathrm{~ms}$.

cavitation behaviours, the order of magnitude of the vapour volume predicted by the simulation remains the same in the three channels.

To try to understand the reason of the coupling at low frequency between the cavitating flows in the different channels, the time evolution of the local flow angle in front of the upper foil was first drawn (Figure 10a). As explained before, the occurrence of large cloud cavitation in Channel 1 corresponds to the increase of the incident flow angle. Contrarily, phases with stable cavitation sheets on the second and third foils correspond to a small flow angle. Longitudinal velocity near the trailing edge, in the boundary layer of the suction side of the upper foil, is drawn Figure 10b with respect to time. It shows that phases of developed cloud cavitation are followed by the passage at the foil trailing edge of travelling vortices, with negative longitudinal velocity. The interactions of these travelling flow structures and the foil trailing edge seem at the origin of the decrease of the flow angle in front of the foil.

Another illustration of this phenomenon is given in Figure 11, where the flow vorticity is drawn at different

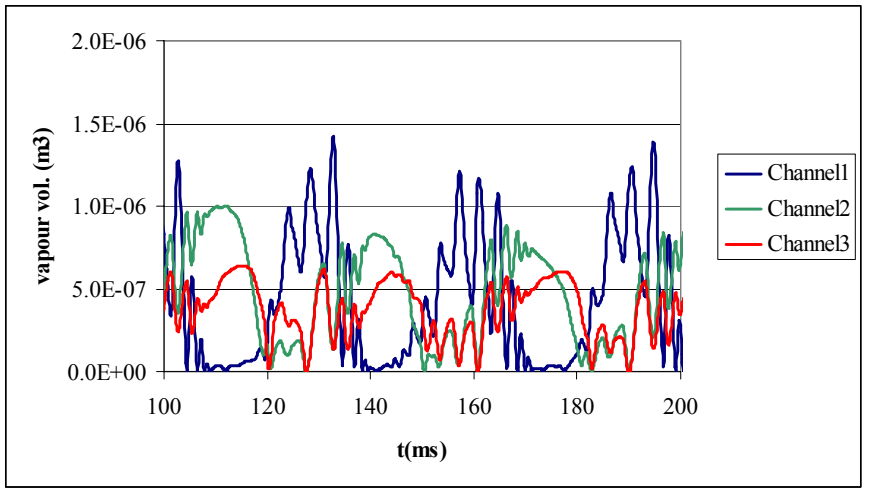

Figure 9. Time evolution of the vapour volume in the three channels. 


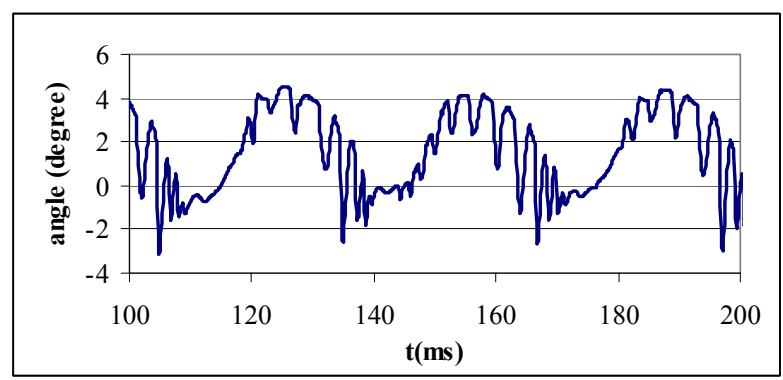

Figure 10a. Angle of velocity vector, $5 \%$ upstream of the upper foil leading edge.
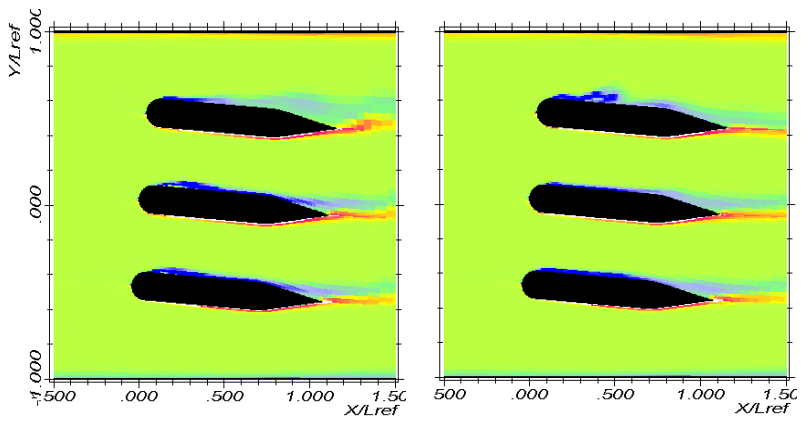

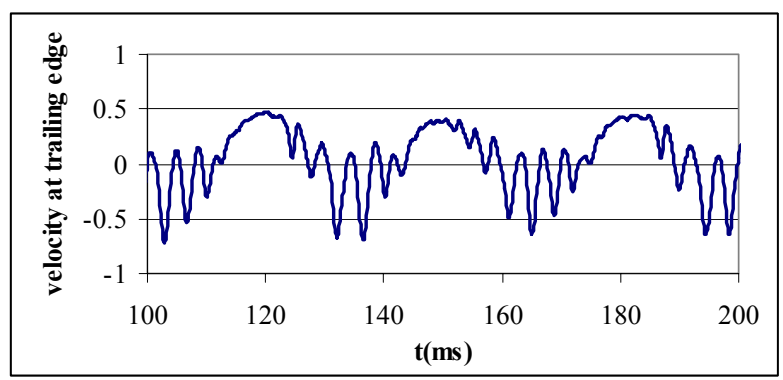

Figure 10b. Longitudinal velocity near the upper foil trailing edge (suction side).
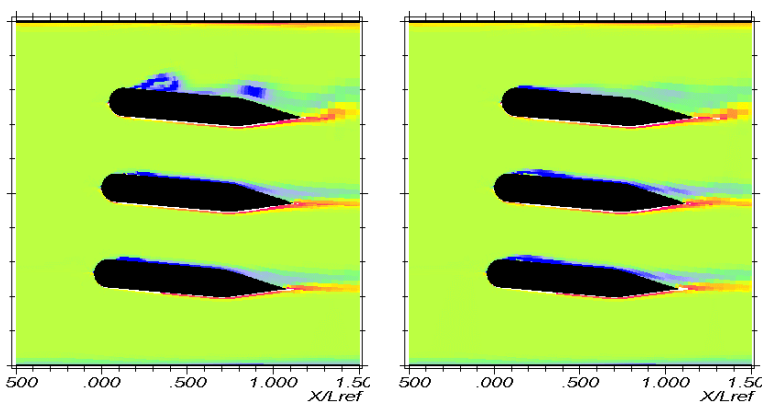

Figure 11. Visualisation of the flow vorticity at $\mathrm{t}=143,153,164$ and $174 \mathrm{~ms}$.

stages of the low frequency cycle. The period of the low frequency behaviour is then certainly linked to the convection time of the large vortices generated by unsteady cavitation, to the foil trailing edge.

\section{Conclusion}

Cavitation was studied in a cascade of three hydrofoils held in a cavitation tunnel. Estimation of the shedding and the coupling frequency was possible only in autocorrelation domain due to the unsteadiness of the cycle duration and the low signal to noise ratio. Filter design on the basis of the frequency determination as it was described above is well suited for the extraction of interesting signal parts. Hilbert transformation and image phase averaging appeared as a useful tool to get detailed information about high speed cyclic processes without the necessity of a high speed camera.

Both the experimental and the numerical investigations show two dominant frequencies. The lower one is about $40 \mathrm{~Hz}$ in the experiments and does not depend on the cavitation number. It is found a little lower $(30 \mathrm{~Hz})$ by the numerical simulation. The higher frequency corresponds to a Strouhal number close to 0.3 for the upper channel, where typical self-oscillating cloud cavitation is observed. At phase angles, when the cavitation in the upper channel is long, the cavitation structures in the other channels are short and vice versa.

According to the phase-averaged videos and the computation results, the lower frequency seems to be strongly linked to the coupling of the three channels. Analyses of numerical results indicate that vortices generated by the cloud cavitation phenomenon in the upper channel are convected by the mean flow to the trailing edge. The flow repartition in the different channels seems strongly affected by the interaction between the travelling vorticies and the trailing edge flow of the upper foil, what may be the origin of the low frequency phenomenon.

The good agreement between experimental and numerical results obtained indicates that the proposed model well describes the unsteady cavitation behaviour and complex associated hydrodynamic effects.

\section{Acknowledgements}

This work is part of a European exchange program PROCOPE with the research teams of Grenoble and Darmstadt as members. The numerical model has been developed with the support of the French space agency CNES. 


\section{References}

Coutier-Delgosha O., Reboud J.L., Albano G. (2000), Numerical simulation of the unsteady Cavitation behavior of an inducer blade cascade, proc. of ASME FEDSM00, Boston, June 2000.

Coutier-Delgosha O., Fortes-Patella, R., Reboud J.L. (2001), Evaluation of the turbulence model influence on the numerical simulations of unsteady cavitation, Proc. of ASME FEDSM01, Cavitation and Multiphase Flow Forum, New Orleans.

Delannoy Y., Kueny JL (1990) Two phase flow approach in unsteady cavitation modelling, Cavitation and Multiphase Flow Forum, ASME-FED vol.98, pp. 153-158

Hofmann M., Lohrberg H., Ludwig G., Stoffel B., Reboud JL., Fortes-Patella R. (1999) Numerical and experimental investigations on the self-oscillating behavior of cloud cavitation: part 1 visualisation / part 2 dynamic pressures, 3rd ASME/JSME Joint Fluids Engineering Conference, July 1999, San Francisco

Hofmann M., Stoffel B.,.Coutier-Delgosha O., Fortes-Patella R., Reboud JL (2001) Experimental and numerical studies an a centrifugal pump with $2 d$-curved blades in cavitating condition, 4th Int. Symp. on Cavitation, Pasadena.

Joussellin F., Courtot Y., Coutier-Delgosha O., Reboud JL. (2001) Cavitating inducer instabilities : experimental analysis and 2D numerical simulation of unsteady flow in blade cascade, 4th Int. Symp. on Cavitation, Pasadena.

Kubota A, Kato H., Yamaguchi H. (1992), A new modelling of cavitating flows: a numerical study of unsteady cavitation on a hydrofoil section, J. Fluid Mech., vol. 240, pp. 59-96.

Reboud J.L. and Delannoy Y. (1994) Two-phase flow modelling of unsteady cavitation, 2nd Int. Symp. on Cavitation, Tokyo, April 94.

Reboud J.L., Stutz B. and Coutier O. (1998). Two-phase flow structure of cavitation : experiment and modelling of unsteady effects, 3rd Int. Symp. on Cavitation, Grenoble, France, April 1998.

Sauer J., and Schnerr G.H. (2000), Unsteady cavitating flow - A new cavitation model based on modified front capturing method and bubble dynamics, Proceedings of FEDSM'00 - Fluids Engineering Summer Conference, Boston, June 2000.

Song and He, Numerical simulation of cavitating flows by single-phase flow approach; 3rd Int. Symp. on Cavitation, Grenoble, France, April 1998.

Zhu J. (1991), A low diffusive and oscillation-free convection scheme, Comm. in Applied Num. Methods, vol. 7. 